\title{
Rapamycin reduces primary effusion lymphoma progression by targeting VEGF production and VEGF responses P Gasperini* and G Tosato
}

Address: Laboratory of Cellular Oncology, Center for Cancer Research, National Cancer Institute, Bethesda, Maryland, USA

* Corresponding author

from I I th International Conference on Malignancies in AIDS and Other Acquired Immunodeficiencies (ICMAOI): Basic, Epidemiologic, and Clinical Research

Bethesda, MD, USA. 6-7 October 2008

Published: 17 June 2009

Infectious Agents and Cancer 2009, 4(Suppl 2):O12 doi:I0.1 186/1750-9378-4-S2-OI2

This abstract is available from: http://www.infectagentscancer.com/content/4/S2/OI2

(C) 2009 Gasperini and Tosato; licensee BioMed Central Ltd.

Kaposi's sarcoma herpesvirus (KSHV)-infected primary effusion lymphoma (PEL) typically presents as a malignant effusion in body cavities that disseminates to distant sites. There is a need for new therapies for PEL as most patients rapidly succumb in spite of high-dose chemotherapy. We examined the potential utility of Rapamycin for PEL treatment. We found that the downstream effectors of the mammalian target of Rapamycin (mTOR) p70S6k and S6 are constitutively phosphorylated in PEL cells, and that Rapamycin inhibits this constitutive p70S6k and S6 phosphorylation. Rapamycin reduces PEL proliferation but is not cytotoxic for PEL cells. Intraperitoneal injection of PEL cells in NOD/SCID mice causes experimental effusion lymphoma. Rapamycin delayed PEL development in this model, markedly reduced accumulation of ascites $(p=0.009)$, prevented formation of solid tumor masses, and a significantly extended mouse survival $(\mathrm{P}<0.001)$. However, Rapamycin did not eradicate PEL in mice. We examined the mechanisms by which Rapamycin reduces PEL progression in this mouse model. Levels of VEGF, which promotes vascular permeability and is critical to the accumulation of body cavity fluids, were significantly reduced in ascites of Rapamycin-treated mice compared to controls $(\mathrm{p}=0.009)$. Rapamycin inhibited VEGF-induced phosphorylation of VEGF receptor (R) 2 in endothelial cells and activation of the downstream effectors of VEGFR2 phosphorylation src and enos (endothelial nitric oxide synthase). Rapamycin did not alter KSHV genes transcription in PEL cells, and only insignificantly reduced levels of IL-10, the principal growth factor for PEL, in ascites of PEL-bearing mice.
Reduction of VEGF secretion by PEL and impairment of endothelial cell responses to residual VEGF likely explain reduced accumulation of ascites in Rapamycin-treated mice. The failure of Rapamycin to significantly reduce IL10 levels in PEL-bearing mice and to promote PEL cell death likely explain PEL persistence in mice treated with Rapamycin. The successful use of Rapamycin to reduce PEL effusion and disease progression by reducing VEGF secretion and endothelial cell responses to VEGF illustrates a novel application of mTOR inhibition that targets the tumor microenvironment rather than the tumor cells, and is applicable to the treatment of PEL and other malignancies characterized by ascites accumulation and increased vascular permeability. 